\title{
Compare the Level of Satisfaction Among Smart Phone and Traditional Hearing Aids Users
}

\author{
Kaleem Abbas, Sadia Nawaz, Affaf Ijaz, Waris Ali, Atia-Ur-Rehman, Hammad Ashraf Mustafai, \\ Humaira Waseem, Baber Ali ,Rimsha Tariq \\ Department of Health Professional Technologies, Faculty of Allied health science, The University of Lahore, \\ Lahore, Pakistan
}

\begin{abstract}
Hearing is crucial for human life, it undertakes a important job in dissertation and linguistic development, which is the fundamental device for the advancement of human correspondence. People with hearing impedance may endure extreme misfortune in their social, mental and proficient lives, dread, misery, disengagement and further more family strains as a result of the absence of consideration influencing those with hearing disorders. Objective: To compare the level of Satisfaction between smart phone hearing aids and traditional hearing aids users. Methods: Cross-sectional study was conducted among Hearing aids users with smart phone hearing aids and traditional hearing aids using purposive sampling technique .SADL (Satisfaction with amplification in daily life) was used to measure the satisfaction level between smart phone hearing aids users and traditional hearing aids users. 100 applicants by moderate to severe senserineural hearing loss of age range from 18 years to 35 years recommended for hearing aids fitting were included in this research by their consent. Data for this research was collected from Sialkot, Lahore, Narowal, Gujranwala, Pakistan. The data of 100 participants were analyzed through SPSS version 25.0 and P-value less than 0.05 was considered significant. Results: A total number of 100 participants were included under which there were 50 males and 50 females.. Out of 100, 53(53\%) participants belong to age group of $(26+35)$ years and $47(47 \%)$ participants belong to age group of $(18+26)$ years. The participants who were using traditional hearing aids, there the level of satisfaction was $48.0 \pm 3.915$ and the level of satisfaction in the participants who were using smart phone hearing aids was $53.95 \pm 4.17$. Smart phone hearing aids users were more satisfied than traditional hearing aids users. Conclusion: It was concluded that smart phone hearing aids users have more satisfaction level as compare to traditional hearing aids users.
\end{abstract}

Keywords: Audiologist, Audible range, Hearing loss, Smart phone Hearing aids, Traditional hearing aids.

DOI: $10.7176 / \mathrm{JHMN} / 69-05$

Publication date: December $31^{\text {st }} 2019$

\section{Introduction:}

Audiology is the profession of specialists in hearing assessment and non-medical management of persons with hearing loss ${ }^{1}$.Hearing loss is the greatest prevalent sensual loss in old people ${ }^{2}$. Here are three types of hearing loss which are as, sensorineural hearing loss, conductive hearing loss and mixed hearing loss ${ }^{3}$.Conventionally, inner ear deformities are supposed to be related with sensorineural hearing loss ${ }^{4}$. Hearing loss can be reduced by using hearing aids ${ }^{5}$.Hearing aid is a stratagem planned to recover hearing by manufacturing sound perceptible to a person with hearing $\operatorname{loss}^{6}$.There stay different types of hearing aids ${ }^{7}$.Smart phone hearing aids are those hearing aids in which a app is used to control the volume and other features of the hearing aids according to condition. Smart phone based hearing aids have more benefits in improving speech as compared to traditional hearing aids users $^{8}$.Gradation of hearing loss dealings and expresses round the particular strictness of the complaint. An individual can be exaggerated in mild hearing loss, moderate, severe and profound levels. Mild hearing loss frequently unspoken allowed but when it arises to moderate level, severe protections are undertaken ${ }^{9}$.Around 5.3\% of the world's population is hearing impaired ${ }^{10}$. Conferring to Pakistan's National Policy for Persons with Disabilities, out of $2.49 \%$ disable population, $7.40 \%$ are deaf ${ }^{11}$. Hearing allow us to part, connect, prepare doings and knowledge the world through listening ${ }^{12}$. Hearing impairment is often related with injury to hair cells in $\operatorname{cochlea}^{13}$. To reduce hearing impairment different kinds of hearing aids are used. The different kinds of hearing aids greatest fitting for an individual rest on on the degree and type of magnification required, ear canal size and form, the structures required ${ }^{14}$.Digital hearing aids are more beneficial as compare to analog hearing aids ${ }^{15}$.Advance technology are smart phone hearing aids which are more useful as compare analog or digital hearing aids ${ }^{16}$. Conductive hearing loss happens when there is a issue shifting sound waves anyplace sideways the trail over outer ear, tympanic membrane or ossicles ${ }^{17}$. Causes of conductive hearing loss are middle ear lesions ${ }^{18}$. If a conductive hearing loss happens in coincidence by a sensorineural hearing loss, it is mentioned to as a mixed hearing loss ${ }^{19}$. Hearing empowers us and assistances us lead our normal exists without limitations ${ }^{20}$.

\section{Methods}

100 applicants with moderate to severe senserineural hearing loss suggested for hearing aids fitting were include in this research by their consent. These applicants was divided into two groups with equal distribution as: Group 
A: were fitted with smart phone hearing aids through which the wearer can control volume and other features. Group B: were fitted with traditional hearing aids. Both groups given the time of two months to use hearing aids. After two months uses a questionnaire SADL (Satisfaction with amplification in daily life) in both groups to find out satisfaction level between smart phone hearing aids users and traditional hearing aids users. After collecting data it was analyzed through SPSS version 25.0.Descriptive statistics were used to analyze variables. Demographics (age , gender ) and to compare the level of satisfaction among smart phone hearing aids users and traditional hearing aids users were analyzed using frequencies, percentage and independent t-test.

\section{Results:}

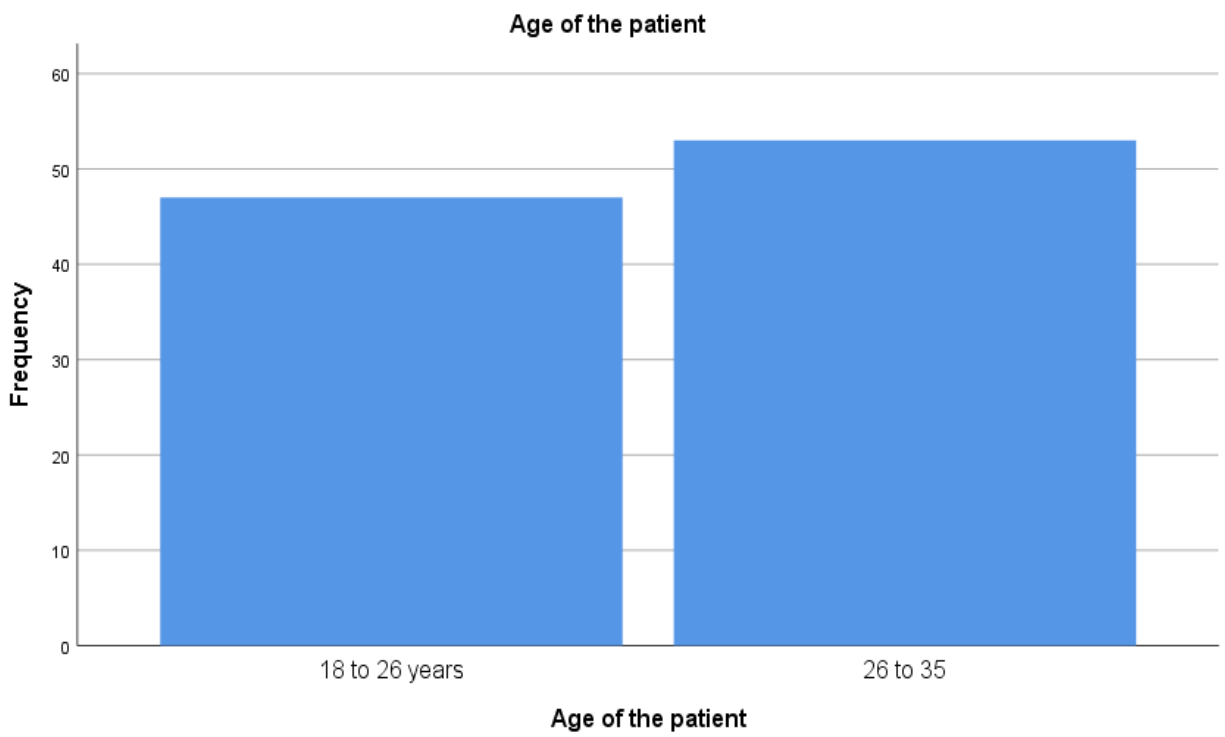

Table 1: Descriptive statistics of gender

A total number of 100 participants were included under which there were 50 males and 50 females. Participants were divided into two groups on the base of age .Out of 100 participants, 53(53\%) participants belong to age group of $(26+35)$ years and $47(47 \%)$ participants belong to age group of $(18+26)$ years.

\begin{tabular}{|l|l|l|l|l|l|}
\hline \multicolumn{5}{|c|}{ group statistics } & P-value \\
\hline \multirow{3}{*}{ Total } & \multicolumn{1}{|c|}{ Category } & \multicolumn{2}{c|}{ Mean \pm S.D } & Std. Error mean & \multirow{2}{*}{0.001} \\
\cline { 2 - 6 } & traditional hearing aids users & 48.00 & 3.91 & .57735 & \\
\hline
\end{tabular}

Table 2: Descriptive statistics of groups

The participants who were using traditional hearing aids there the level of satisfaction was $48.0 \pm 3.91$ and the level of satisfaction in the participants who were using smart phone hearing aids was $53.95 \pm 4.17$. There was significance difference between the traditional hearing aids users and smart phone hearing aids users. Smart phone hearing aids users were more satisfied than traditional hearing aids users. Finding indicates that the level of satisfaction in smart phone hearing aids users were more as compared to the level of satisfaction in traditional hearing aids users.

\section{Discussion}

The aim of study was to equate the level of satisfaction between smart phone hearing aids users and traditional hearing aids users. This study was carried out in Narowal, Gujranwala, Lahore, Sialkot, Pakistan which includes the participants who were the candidates of hearing aids fitting. In this study 100 applicants with moderate to severe senserineural hearing loss suggested for hearing aids fitting were include in this research by their consent. These applicants were divided into two groups with equal distribution as: Group A: were fitted with smart phone hearing aids through which the wearer can control volume and other features. Group B: were fitted with traditional hearing aid. Both groups given the time of two months to use hearing aids. After two months uses a questionnaire SADL (Satisfaction with amplification in daily life) in both groups to find out satisfaction level between smart phone hearing aids users and traditional hearing aids users. Out of 100 participants, 53(53\%) participants belong to age group of $(26+35)$ years and $47(47 \%)$ participants belong to age group of $(18+26)$ years. The level of satisfaction in smart phone hearing aids users was 53.95 \pm 4.17 and the level of satisfaction in traditional hearing aids users was $48.0 \pm 3.915$. The conclusion specifies that satisfaction levels in contributors using smart phone 
hearing aids were extremely significant as compared to the participants using traditional hearing aids. While the majority reported in previous researches shows that the satisfaction with the hearing aids increased when it provides smart phone hearing aids to the participants using hearing aids ${ }^{21}$.SADL (Satisfaction with Amplification in Daily Life) a standard questionnaire which was used to compare the satisfaction level between the smart phone hearing aids users and traditional hearing aids users ${ }^{22}$.A study was conducted by AM Amlani, B Taylor, C Levy as Utility of smartphone-based hearing aid applications as a substitute to traditional hearing aids and there results indicates that the satisfaction level in smart phone hearing aids users was more than traditional hearing aids users $^{23}$. Similarly, in our study the level of satisfaction among smart phone hearing aids users was more as compared to traditional hearing aids users. A study was conducted in January 2010 by Kochkin, Sergei as Marke Trak VIII Consumer satisfaction with hearing aids is slowly increasing ${ }^{24}$.Digital and smart phone hearing aids have more benefits according to satisfaction level ${ }^{25}$.

\section{Conclusion}

This study concluded that there was more satisfaction level in smart phone hearing aids users as compared to the level of satisfaction in traditional hearing aids users. Our research results indicated that smart phone hearing aids technology are most advanced technology and hearing aids users gives more preference to smart phone hearing aids technology as compared to traditional hearing aids technology. Smart phone hearing aids has more benefits as compared to traditional hearing aids.

\section{References}

1. Hall JW. Introduction to audiology today: Pearson Boston, MA 2014.

2. Roth TN, Hanebuth D, Probst R. Prevalence of age-related hearing loss in Europe: a review. European Archives of Oto-Rhino-Laryngology. 2011;268(8):1101-1107.

3. Bosmana AJ, Smoorenburg GF. Intelligibility of Dutch CVC syllables and sentences for listeners with normal hearing and with three types of hearing impairment. Audiology. 1995;34(5):260-284.

4. Johnson J, K. Lalwani A. Sensorineural and conductive hearing loss associated with lateral semicircular canal malformation. The Laryngoscope. 2000;110(10):1673-1679.

5. LANTANG KV. EFFECT OF ASSISTIVE TECHNOLOGY IN ENHANCING ACADEMIC PERFORMANCE OF STUDENTS WITH HEARING IMPAIRMENT IN BUEA.

6. Meyer C, Hickson L. What factors influence help-seeking for hearing impairment and hearing aid adoption in older adults? International journal of audiology. 2012;51(2):66-74.

7. Dillon H. Hearing aids: Hodder Arnold 2008.

8. Abu-Ghanem S, Handzel O, Ness L, et al. Smartphone-based audiometric test for screening hearing loss in the elderly. European archives of oto-rhino-laryngology. 2016;273(2):333-339.

9. Cole EB, Flexer C. Children with hearing loss: Developing listening and talking, birth to six: Plural Publishing 2019.

10. Exeter DJ, Wu B, Lee AC, et al. The projected burden of hearing loss in New Zealand (2011-2061) and the implications for the hearing health workforce. The New Zealand Medical Journal. 2015;128(1418):12.

11. Akram B, Bashir R. Special Education and Deaf Children in Pakistan: An Overview. Journal of Elementary Education. 2012;22(2):33-44.

12. Wallhagen MI. The stigma of hearing loss. The Gerontologist. 2009;50(1):66-75.

13. Moore BC. Dead regions in the cochlea: Diagnosis, perceptual consequences, and implications for the fitting of hearing aids. Trends in amplification. 2001;5(1):1-34.

14. Hampson R. Hearing aids. European Geriatric Medicine. 2012;3(3):198-200.

15. Kaplan-Neeman R, Muchnik C, Hildesheimer M, et al. Hearing aid satisfaction and use in the advanced digital era. The Laryngoscope. 2012;122(9):2029-2036.

16. Paglialonga A, Tognola G, Pinciroli F. Apps for hearing science and care. American Journal of Audiology. 2015;24(3):293-298.

17. Mikulec AA, McKenna MJ, Ramsey MJ, et al. Superior semicircular canal dehiscence presenting as conductive hearing loss without vertigo. Otology \& Neurotology. 2004;25(2):121-129.

18. Merchant SN, Rosowski JJ. Conductive hearing loss caused by third-window lesions of the inner ear. Otology \& neurotology: official publication of the American Otological Society, American Neurotology Society [and] European Academy of Otology and Neurotology. 2008;29(3):282.

19. Baumgartner W-D, Böheim K, Hagen R, et al. The vibrant soundbridge for conductive and mixed hearing losses: European multicenter study results. Active Middle Ear Implants: Karger Publishers 2010:38-50.

20. Folmer RL. The importance of hearing conservation instruction. The Journal of School Nursing. 2003;19(3):140-148.

21. Aldaz G, Puria S, Leifer LJ. Smartphone-based system for learning and inferring hearing aid settings. Journal of the American Academy of Audiology. 2016;27(9):732-749. 
22. Cox R, Alexander G. Satisfaction with amplification in daily life. 1999

23. Amlani AM, Taylor B, Levy C, et al. Utility of smartphone-based hearing aid applications as a substitute to traditional hearing aids. The Hearing Review. 2013;20(13):16-18.

24. Kochkin S. MarkeTrak VIII: Consumer satisfaction with hearing aids is slowly increasing. The Hearing Journal. 2010;63(1):19-20.

25. Walden BE, Surr RK, Cord MT, et al. Comparison of benefits provided by different hearing aid technologies. Journal of the American Academy of Audiology. 2000;11(10):540-560. 